\title{
Atrial functional and geometrical remodeling in male highly trained athletes - for better or
}

worse?

Luigi Gabrielli ${ }^{1,2}$; Bart H Bijnens ${ }^{3,4}$; Constantine Butakoff, ${ }^{3}$; Nicolas Duchateau, ${ }^{1}$; Silvia Montserrat, ${ }^{1}$; Beatriz Merino ${ }^{1}$; Josep Gutierrez ${ }^{5}$; Carles Paré ${ }^{1}$; Lluis Mont ${ }^{1}$; Josep Brugada ${ }^{1}$; Marta Sitges ${ }^{1}$.

1 Thorax Clinic Institute, Hospital Clínic, Institut d'Investigacions Biomèdiques August Pi i Sunyer, University of Barcelona, Spain.

2 Cardiovascular Disease Division, Pontificia Universidad Católica de Chile, Santiago, Chile.

3 Universitat Pompeu Fabra, Barcelona, Spain.

4 ICREA (Institució Catalana de Recerca i Estudis Avançats), Barcelona, Spain.

5 Consell Catala de l'Esport, Barcelona, Spain.

Corresponding authors: $\quad$ Marta Sitges, $\mathrm{MD}, \mathrm{PhD}$

Phone \# 34- 93- 2279305

Fax \# 34- 93- 4514148

E-mail: msitges@clinic.ub.es

Luigi Gabrielli, MD

Phone \# 34- 93- 2279305

Fax \# 34- 93- 4514148

E-mail: lgabrielli@med.puc.cl

Word count: 3395 


\begin{abstract}
Purpose: Highly trained athletes have an increased risk of atrial arrhythmias. Atrial geometrical and functional remodeling may be the underlying substrate. We analyze and relate atrial size, deformation and performance in professional handball players compared with non-sportive subjects.
\end{abstract}

Methods: 24 professional handball players and 20 non-sportive males were compared. All subjects underwent an echocardiographic study with evaluation of left (LA), right atrial (RA) dimensions and deformation by strain (Sa) and strain rate (SRa). Atrial performance was assessed from the atrial stroke volume (SV). With computational geometrical models, we studied the relation between atrial volumes, strains and SV and compared atrial working conditions. We estimated the functional reserve and a resulting average wall stress.

Results: LA and RA volumes were larger in athletes than in controls $(35.2 \pm 8.8$ vs $24.8 \pm 4.3 \mathrm{ml} / \mathrm{m}^{2}, \mathrm{p}<0.01$ and $29.0 \pm 8.4$ vs $19.0 \pm 5.1 \mathrm{ml} / \mathrm{m}^{2}, \mathrm{p}<0.01$ respectively). LASa and RASa during active atrial contraction were decreased in athletes $(-12.2 \pm 2.0$ vs $-14.5 \pm 2.1 \%, \mathrm{p}<0.01$ and $12.1 \pm 1.8$ vs $-14.2 \pm 1.5 \%, \mathrm{p}<0.01$ respectively). LASV was similar between groups $(6.6 \pm 1.4 \mathrm{vs}$ $7.3 \pm 1.1 \mathrm{ml}, \mathrm{p}=0.19)$ and RASV was lower in athletes $(6.2 \pm 1.3$ vs $7.2 \pm 1.1 \mathrm{ml}, \mathrm{p}<0.01)$. Computational models showed that this different operational mode potentially increases performance reserve, but at the cost of higher atrial wall stress.

Conclusion: a proportion of athletes with enlarged LA and RA showed different atrial contractile performance, likely resulting in atria working at higher wall stress.

Key words: atrial strain; atrial function; echocardiography; wall stress; exercise. 
Abbreviations

$\mathrm{AF}$, atrial fibrillation

CMR, cardiac magnetic resonance

LA, left atrium

LASa, peak negative left atrial strain during active atrial contraction

LASRa, peak negative left atrial strain rate during active atrial contraction

LASRe, LA early diastolic peak strain rate

LASRs, LA peak strain rate during ventricular systole

LASs, LA peak strain during ventricular systole

LV, left ventricle

$\mathrm{RA}$, right atrium

RASa, peak negative right atrial strain during active atrial contraction

RASRa, peak negative right atrial strain rate during active atrial contraction

RASRe, RA early diastolic peak strain rate

RASRs, RA peak strain rate during ventricular systole

RASs, RA peak strain during ventricular systole

$\mathrm{RV}$, right ventricle

$\mathrm{SV}$, stroke volume 


\section{Introduction}

Observational studies have reported an increased prevalence of atrial fibrillation (AF) in highly trained athletes, as compared to case-matched controls from the general population [Elosua et al. 2009 and Molina et al. 2008]. Particularly, a cohort of retired handball players showed an AF incidence of $30 \%$ at the age of 50 [Van Buuren F et al. 2012]. A meta-analysis of these observational studies showed a five-fold increase in the overall risk of AF in this population [Abdulla J and Rokkedal J. 2009]. The etiology of AF in this particular group of subjects is still poorly understood and several theories have been suggested [Turagam $M$ et al. 2012]. Increased vagal tone with consequent bradycardia may lead to dispersion of atrial repolarization, which in turn may increase the susceptibility to AF [Wilhelm M et al. 2011]. The remodeling process induced in the atria by intensive exercise, together with the above factors, may translate into a potential dysfunction [Pellicia A et al. 2005], and lead to the development of the arrhythmia. Atrial flutter can also be present or co-exist with AF and has been linked to right atrial dilatation in the setting of the athlete's heart [Erol MK and Karakelleoglu S. 2002].

In recent years, atrial strain and strain-rate analysis by two-dimensional speckle tracking has emerged as a novel method to evaluate left atrial (LA) and right atrial (RA) function [Padelleti M el al. 2012; Saraiva RM et al. 2010]. The assessment of LA geometry [Tsang MY et al. 2012], and function by strain and strain-rate has been used as a predictor of the occurrence of AF in different clinical scenarios such as pulmonary veins ablation [Schneider C et al. 2008], and coronary artery by-pass grafting [Gabrielli L et al. 2009]. Additionally, it was shown that increased local wall stress is associated with focal atrial remodeling and electrical alterations [Hunter RJ et al. 2012]. 
Simple computational models can help understanding the link between chamber size, deformation and contractile performance [Bijnens BH et al. 2012]. In the present study, we apply an ellipsoid-based computational model to the data collected from a set of athletes and control subjects, in order to reach a better understanding of how these variables interrelate in these populations. This model has been previously used to assess ventricular wall stress and to perform a detailed analysis of ventricular performance [Mirsky et al. 1981]. The objective of the present study is to provide insights into the atrial remodeling process in athletes; our hypothesis is that a subgroup of athletes might have different atrial working conditions that might favour the development of a potential arrhythmogenic substrate.

\section{Methods}

\section{Study design and population}

This was a cross-sectional study, comparing two groups: 24 athletes and 20 sedentary healthy controls with similar age who were recruited among hospital staff and patients' relatives with a strictly normal medical record. All participants were male, in sinus rhythm, normotensive, did not take any medication or anabolic drug and did not have other cardiovascular disease or risk factors. The athletes group consisted of professional handball players from the same national team. All of them had been training an average of 15 hours/week during the past year. All subjects in the control group did not participate in routine competitive or recreational sports.

The study was approved by the ethics committee of our institution and informed consent was obtained from all individuals. 


\section{Echocardiography}

Each individual underwent a complete two-dimensional echocardiographic study using a commercially available ultrasound scanner (Vivid Q, GE Healthcare, Milwaukee, WI) with a 2.5MHz phased array transducer (M4S). Standard echocardiographic views were obtained. Images were analyzed off-line with a commercially available software (EchoPac version 108.1.6, GE Healthcare). Left ventricle (LV) and right ventricle (RV) measurements were done according to the recommendations of the American Society of Echocardiography [Lang RM et al. 2006]. LA volume was calculated from apical four and two-chamber views using the biplane method of discs and RA volume was calculated from a monoplane derived volume (apical four chamber view). LA and RA emptying fractions were calculated using the difference between the maximal volumes (just before the opening of the mitral valve) and the minimal ones (at the closure of the mitral valve) / maximal volume. Atrial active stroke volume (SV) was estimated using the difference between the pre-A volume (obtained from the last frame before mitral valve opening or at time of $\mathrm{P}$ wave on the surface electrocardiogram) and minimal volume, Also, from the measured atrial volumes we calculated LA/RA volumes of early diastolic phase (maximal volume - pre-A volume) and LA/RA emptying fractions of early diastolic phase ([maximal volume - pre-A volume]/maximal volume). Area and volume measurements were indexed by body surface area to allow proper comparison between groups and all data were analyzed by two blinded, experienced sonographers.

To define severe atrial dilatation, a cut-off of $40 \mathrm{ml} / \mathrm{m}^{2}$ was used for both atria [Lang RM et al. $2006]$.

\section{Atrial strain and strain rate}


Strain and strain rate of both atria were analyzed off-line with a commercially available software package (2Dstrain, EchoPac version 108.1.6, GE Healthcare). Acquisitions were performed with an optimazed image with a frame rate of at least $50 \mathrm{fps}$ and with focal point in atriums. The endocardial border was manually traced using a point-and-click technique. LA and RA strains were calculated with the reference point set at the onset of the P-wave of the surface ECG, which allowed identifying the peak negative strain (shortening) during atrial contraction (LASa and RASa, respectively) Similarly, the peak negative strain rate (active shortening) during atrial contraction was identified (LASRa and RASRa, respectively). The software divided the atrial wall into 6 segments and the average was taken for analysis (Figures 1A and 1B). Also, LA/RA peak strain and strain rate during ventricular systole and strain rate during early diastolic phase were recorded.

\section{Computational geometrical model}

The possible relations between atrial volume (pre-A volume, taken before the onset of atrial contraction), the global atrial longitudinal strain during active contraction, and the SV generated by the atrial contraction, were investigated with a simple geometrical mathematical model.

In this model, the atria were approximated by ellipsoids with two of the (short) radii being equal and with long axis / short axis ratios of 1.26 (left atrium) and 1.16 (right atrium), corresponding to the average values measured from our data. In order to simulate the potential range within which the atria of the studied subjects could function, we calculated the volume-strain-SV relations for a pre-A volume from 18 to $95 \mathrm{ml}$ (for the LA) or 19 to $105 \mathrm{ml}$ (for the RA), and for a SV from 3.5 to $10 \mathrm{ml}$ (for both atria). The global strain during atrial contraction was calculated from the pre-A and minimal volumes based on the radius change during atrial contraction. Global 
atrial longitudinal strain was computed as the change in the largest circumference, whose formula is detailed in Appendix.

Next, in order to find the (resting) working point in this volume-strain-SV relation for each individual from our study, the ellipsoid model was fitted on each of the individual's measured atrial volumes and diameters, as also detailed in Appendix. This personalized model provided an estimation of the SV reserve and a rough approximation of average atrial wall stress for each individual.

\section{Statistical methods}

Continuous baseline variables were expressed as mean \pm standard deviation (SD) values or median (interquartile range), after checking for normal distribution as assessed by the Kolmogorov-Smirnov test. Categorical variables were expressed as total number (percentages) and compared between groups using Chi-square or Fisher's test when appropriate. Continuous variables were tested by unpaired $t$ test or Mann-Whitney $U$ test, according to normality, and paired data by paired t test or Wilcoxon analysis. Pearson or Spearman methods were used to analyze the correlation between continuous variables when suitable. Reproducibility of the observations was assessed offline using Bland-Altman analysis on 10 randomly selected subjects, from 2 different times (intra-observer) and by 2 different observers (inter-observer). The acquisitions were performed by one, blind expert operator and all the measurements were performed from the same acquisition. Statistical significance was established at $\mathrm{P}<0.05$. All data were analyzed using the SPSS statistical package version 15.0 (SPSS Inc., Chicago, IL). 


\section{Results}

\section{Population characteristics}

The clinical characteristics of the studied population are listed in Table 1. All participants were male. Body mass index and body surface area were significantly higher in athletes than in controls. In all subjects, no significant mitral or tricuspid regurgitation were found.

Table 2 shows the characteristics of LA and LV in athletes and controls. LV dimensions and function were similar in both groups while LV mass was higher in athletes. LA maximum volume was increased in athletes as compared to controls, LA emptying fraction and active LA SV (both volumetric and Doppler-based) were similar between both groups and athletes showed reduced LASa and LASRa as compared to controls.

Table 3 shows RV and RA dimensions and function. Athletes had increased RV basal diameter as compared to controls and RV function was similar between groups. In athletes, RA maximum volume was increased and RASa and RASRa were reduced as compared to controls.

We did not find differences in the other phases of LA/RA cycle regarding emptying fraction and deformation (Table 2 and 3).

\section{Atrial size, deformation and contractile performance}

Figures $2 \mathrm{~A}, 2 \mathrm{~B}$ and $2 \mathrm{C}$ show the relation between the active LA deformation and the maximal LA atrial size, for all individuals. A proportion of athletes (circle in the figure) showed a larger LA volume, lower deformation (LASa and LASRa) and lower LA SV during active atrial contraction: athletes with LA volume $\geq 40 \mathrm{ml} / \mathrm{m}^{2} \quad(\mathrm{n}=7)$ showed significantly reduced LASa, LASRa and active LA SV during atrial contraction as compared to athletes with LA volume $<40$ 
$\mathrm{ml} / \mathrm{m}^{2}\left(-10.33 \pm 1.61 \%\right.$ vs $-13.03 \pm 2.01 \%, \mathrm{p}<0.01[\mathrm{LASa}] ;-1.08 \pm 0.12 \mathrm{~s}^{-1}$ vs $-1.70 \pm 0.48 \mathrm{~s}^{-1}$, $\mathrm{p}=0.01[\mathrm{LASRa}]$ and $5.2 \pm 1.1 \mathrm{ml}$ vs $7.2 \pm 1.5 \mathrm{ml}, \mathrm{p}<0.01$ [LA SV]).

Similarly to what was reported for the LA, Figures $2 \mathrm{D}, 2 \mathrm{E}$ and $2 \mathrm{~F}$ show the relation between RA maximal volume and RASa and RA SV. A proportion of athletes (circle in the figure) also showed a larger RA volume, lower deformation (RASa and RASRa) and lower RA SV during active atrial contraction, and similarly to what was observed with LA: athletes with a RA volume $\geq 40 \mathrm{~mL} / \mathrm{m}^{2}(\mathrm{n}=4)$ showed significantly reduced RASa, RASRa and RA SV during active atrial contraction as compared to athletes with RA volume $<40 \mathrm{~mL} / \mathrm{m}^{2}(-9.55 \pm 0.92 \%$ vs $-12.63 \pm$ $1.76 \%, \mathrm{p}<0.01[\mathrm{RASa}] ;-0.99 \pm 0.07 \mathrm{~s}^{-1} \mathrm{vs}-1.38 \pm 0.53 \mathrm{~s}^{-1}, \mathrm{p}=0.04$ [RASRa] and $4.7 \pm 0.7 \mathrm{~mL}$ vs $6.5 \pm 1.2 \mathrm{~mL}, \mathrm{p}=0.01$ [RA SV]). All athletes with severe RA dilatation showed severe LA dilatation.

Among athletes there was a significant correlation between LASa with LA maximal volume $(\mathrm{r}=$ 0.57, $\mathrm{p}<0.01)$, with LASRa $(\mathrm{r}=0.73, \mathrm{p}<0.01)$ and with LA SV $(\mathrm{r}=-0.63, \mathrm{p}<0.01)$; and between RASa with RA maximal volume $(r=0.64, \mathrm{p}<0.01)$ with $\operatorname{RASRa}(r=0.62, \mathrm{p}<0.01)$ and with RA SV $(r=-0.78, p<0.01)$.

Finally, athletes showed a significant correlation between LASa and RASa and between LA and RA volume $(\mathrm{r}=0.50, \mathrm{p}=0.017$ [strain]; $\mathrm{r}=0.73, \mathrm{p}<0.01$ [volumes]), indicating a similar performance/remodeling of both atria in a given athlete.

\section{Atrial strain and strain rate reproducibility}

The inter-observer and intra-observer agreement for LA and RA strain and strain rate are detailed in Table 4. In summary, the inter-observer variability was $<6 \%$ and the intra-observer variability was $<2 \%$. 


\section{Geometrical modeling}

As commented in the Methods section, an ellipsoid-based computational model was used for (i) interpreting of the relation between the above-measured parameters (atrial size, deformation and contractile performance), and (ii) for investigating the link between atrial SV reserve, wall stress, and volume.

Figure 3 shows the expected (geometrical) relations between deformation, size and SV (solid lines) for different combinations of pre-A volume and SV in the LA and RA. The working point of each of individual (athletes and controls) was specified (colored dots). The figure indicates that individuals with larger volumes need a lower strain to maintain a similar SV. Additionally, while several athletes were located among the control subjects, a subset of athletes had larger atria with lower strain and SV. Observations were comparable for both left and right atria.

Figure 4 represents the predicted atrial SV reserve (top), the maximal atrial wall stress (middle) and the atrial wall stress associated with a predicted atrial SV reserve (bottom), for each of the individuals in the study. Note that these data results from the personalization of the model to each individual's data, as commented in the Methods section. As it can be observed, a majority of the athletes had a larger SV reserve because of working with larger atria (and therefore lower strain at rest, as shown in figure 3). However, this comes at the cost of elevated wall stress. Additionally, different subgroups of athletes may be considered. The ones with large atrial volumes present a high potential to increase the SV, but at the cost of a high wall stress. The second subgroup has a size similar to controls, with a moderate potential to increase SV, but with apparently higher wall stress than controls because of a different aspect ratio of the atrium. Observations were comparable for both left and right atria. 


\section{Discussion}

Our study first showed that, in a population of young athletes (professional elite handball players), both atria have larger volumes and reduced deformation during active atrial contraction at rest as compared to controls, and a subgroup of athletes has significantly higher volumes and lower deformation. Then, the use of an ellipsoid-based computational model allowed understanding the different working conditions of these subgroups. Larger volumes provide an acute improved performance (by requiring lower deformation to maintain a similar stroke volume) and reserve, but at the cost of higher wall stress. Our interpretation is that this might lead to long term damage, which could explain the increased incidence of atrial arrhythmia observed in this population.

Structural remodeling is seen as the main contributor for initiation and persistence of AF or atrial flutter [Sitges $M$ et al. 2009]. It has been shown that the LA may be enlarged in as many as $20 \%$ of competitive athletes [Pelliccia A et al. 2005], but not all of these subjects developed AF. Indeed, other mechanisms, besides increased atrial size, have been proposed to explain the development of an arrhythmia, such as increased parasympathetic tone, reduced sympathetic tone and increased inflammation [Sorokin AV et al. 2011].

While LA enlargement is common in highly trained athletes, it is suggested that this represents a physiologic adaptation to exercise and chronic training and not a consequence of increased left ventricular filling pressures secondary to diastolic dysfunction [D'Andrea A et al. 2010]; accordingly, the risk of atrial arrhythmias is mildly elevated and developed later in life [Molina L et al 2008; Van Buuren F et al 2012]. Our results confirm that resting LV filling pressures on the 
basis of E/e' ratio and diastolic function are not different in these highly trained athletes and the control group.

In an animal model of chronic endurance training, it was demonstrated that additionally to atrial enlargement, collagen content and myocardial fibrosis markers were significantly increased in both atria after training; these histological and molecular findings were observed together with an increased inducibility of atrial arrhythmias [Benito B et al. 2011]. In the present study we could see that, while a majority of athletes showed atrial properties similar to controls, there was a subgroup presenting marked bi-atrial remodeling. These athlete's atria are working at lower strain and larger volumes for a given or lower, atrial stroke volume as compared to controls. While this might be beneficial to have a larger SV reserve (since when increasing the strain, with a larger atrium they could generate much more SV), it would also result in higher wall stress. Since there is an intrinsic relation between size, deformation and $\mathrm{SV}$, and that individuals can perform at different 'working points', this does not necessarily imply that larger dilatation is a synonym for atrial dysfunction (it is unclear whether this dilatation is indeed physiological). It could be the result of having to cope with the higher SV demand in better performing athletes, or it could also be dilatation and the decrease in deformation and contractility as a result of ongoing atrial wall damage, similar to what can be seen in severe valve regurgitation [Margreet A et al. 2010], or other overload situation [Marciniak A et al. 2010]. Nonetheless, it is clear that the elevated wall stress is an important risk factor for the initiation/worsening of myocardial damage, and that on the longer run, this will result in increased vulnerability to atrial arrhythmias [Di Martino E et al. 2011; Tsai CT et al. 2011].

Consequently, in order to determine whether the atria in an individual are dysfunctional, a more profound analysis of deformation, size and performance, and especially the response to exercise 
(testing if the predicted SV increase can indeed be realized), together with some kind of tissue characterization (e.g. using CMR delayed enhancement), is required [Prinz C et al. 2012]. This means that the understanding of the direct link between atrial remodeling and the risk of arrhythmia in athletes still needs to be improved.

In our study we also discovered a significant correlation between LASa and RASa showing that both atria of a given individual receive similar remodeling triggers, which also could explain the concomitant occurrence of LA and RA arrhythmias in those athletes with pathologic atrial remodeling [Hoogsteen J et al. 2004].

Regarding the other phases of the atrial cycle we did not find differences in terms of emptying fraction and deformation in agreement with other previous report [Gabrielli et al. 2012].

One limitation of our work is that our model only provides an average approximation of atrial wall stress based on global morphologic characteristics. Given the complex shape of the atria, a 3D localized approach would be needed to get accurate estimates of wall stress. However, the estimated values are in range of what has been published before for a detailed analysis, and since we are only interested in the tendency for wall stress rather than the exact values or the individual comparison, they provide an acceptable first approximation. The model-based calculation of SV reserve assumes a normal, physiologic response to exercise and does not include abnormal or pathological changes in atrial function (e.g inability to increase deformation and thus SV). While the precision of such a modeling might be discussed, this approximation allows us to identify subgroups with different behaviors. Other important limitations are the small sample size and the fact that these findings were not extended to other sports disciplines or to a female population.

Current speckle tracking tools may perform worse when applied to the right side of the heart and the atria, due to the potential lower quality of the images (specially considering the worse lateral 
resolution in 2-dimensional echocardiography) and the fact that the algorithms are developed for the left ventricle. However, we believe these measurements are reliable, because an adequate endocardial tracking is ensured and checked frame-by-frame and deformation values are in keeping with what is seen in the image.

Finally, athlete's atrial volumes are not particularly high (D'Andrea et al. 2013) but we used the proposed cut off value of $40 \mathrm{ml} / \mathrm{m}$ proposed by guidelines as severe dilatation (Lang et al. 2006) to separately analyze extreme values of atrial size.

Even considering all the limitations, we believe that our findings, especially given the highly comparable group of individuals, are robust enough to generate a hypothesis to be confirmed in larger studies and including a more elaborated analysis of atrial function, in particular during exercise.

\section{Conclusion}

To our knowledge this is the first study that assessed active atrial function in highly trained athletes and showed that some of these show remodeling that is suggestive of atrial dysfunction. Why some athletes from the same team, with similar training and thus comparable cardiac overload, show completely different adaptation/remodeling, remains an unanswered question and might be related to other predisposing factors that increase the risk of pathologic atrial remodeling and increase the vulnerability to atrial arrhythmias. 


\section{Acknowledgements}

This study was partially supported by grants from the "Generalitat de Catalunya (Consell Catala de l'Esport)", from "Grupo Memora (SFB-Grupo Memora)", Spanish Society of Cardiology (Spanish Heart Foundation), from the "Plan Nacional I+D+I", Spanish Government (DEP201020565), and from the "Subprograma de Proyectos en Salud", Instituto de Salud Carlos III, Spanish Government (FIS - PI11/01709).

Authors have nothing to disclose 


\section{References}

1. Abdulla $\mathbf{J}$ and Rokkedal $\mathbf{J}$ (2009). Is the risk of atrial fibrillation higher in athletes than in the general population? A systematic review and meta-analysis. Europace 11: 1156-1159.

2. Benito B, Gay-Jordi G, Serrano-Mollar A et al (2011). Cardiac arrhythmogenic remodeling in a rat model of long-term intensive exercise training. Circulation 123:13-22.

3. Bijnens BH, Cikes M, Butakoff C, Sitges M, Crispi F (2012). Myocardial motion and deformation: What does it tell as and How does it relate to function?. Fetal Diag Ther $32: 5-16$

4. D'Andrea A, Riegler L, Cocchia R et al (2010). Left atrial volume index in highly trained athletes. Am Heart J 159:1155-61.

5. D'Andrea, Riegler L, Rucco MA et al (2013). Left atrial volumen index in healthy subjects: clinical and echocardiographic correlates. Echocardiography 30:1001-7.

6. Di Martino E, Bellini C, Schwartzman D (2011). In vivo porcine left atrial wall stress: Effect of ventricular tachypacing on spatial and temporal stress distribution. Journal of Biomechanics 44:2755-2760.

7. Elosua R, Arquer A, Mont L, García-Moran E, Brugada J, Marrugat J (2006). Sport practice and the risk of lone atrial fibrillation: a case control study. Int J Cardiol 108:3327.

8. Erol MK, Karakelleoglu S (2002). Assessment of right heart function in the athletes heart. Heart Vessels 16:175-80.

9. Falsetti HL, Mates RE, Grant C, Greene DG, Bunnell IL (1970). Left ventricular wall stress calculated from one - plane cineangiography. Circ Res 26:71-83. 
10. Gabrielli L, Corbalan R, Córdova S et al (2011). Left Atrial Dysfunction Is a Predictor of Postcoronary Artery Bypass Atrial Fibrillation: Association of Left Atrial Strain and Strain Rate Assessed by Speckle Tracking. Echocardiography 28:1104-8.

11. Gabrielli L, Enríquez A, Córdova S, Yañez F, Godoy I, Corbalán R (2012). Assessment of left atrial function in hypertrophic cardiomyopathy and athlete's heart: a left atrial myocardial deformation study. Echocardiography 29:943-9.

12. Hoogsteen J, Schep G, Van Hemel NM, Van Der Wall EE (2004). Paroxysmal atrial fibrillation in male endurance athletes. A 9 years follow up. Europace 6: 222-8.

13. Hunter RJ, Liu Y, Lu Y, Wang W, Schilling RJ (2012). Left atrial wall stress distribution and its relationship to electrophysiologic remodeling in persistent atrial fibrillation. Circ Arrhythm Electrophysiol 5:351-60.

14. Lang RM, Bierig M, Devereux RB et al (2006). Recommendations for chamber quantification. Eur J Echocardiogr 7:79-108.

15. Marciniak A, Claus P, Sutherland GR et al (2007). Changes in systolic left ventricular function in isolated mitral regurgitation. A strain rate imaging study. Eur Heart J 28:26272636.

16. Margreet A, Maass A, Oberdorf-Maass S, Van Veldhuisen D, Van Gilst W, Van Gelder I (2010). Mechanisms of atrial structural changes caused by stretch occurring before and during early atrial fibrillation. Cardiovascular Research 89:754-765.

17. Mirsky I, Krayenbuehl HP (1981). The role of wall stress in the assessment of ventricular function. Herz 6:288-99.

18. Molina L, Mont L, Marrugat J et al (2008). Long term endurance sport practice increases the incidence of lone atrial fibrillation in men: a follow up study. Europace 10: 618-23. 
19. Padeletti M, Cameli M, Lisi M, Malandrino A, Zacá V, Montillo S (2012). Reference values of right atrial longitudinal strain imaging by two-dimensional speckle tracking. Echocardiography 29:147-52.

20. Pelliccia A, Maron BJ, Di Paolo FM et al (2005). Prevalence and clinical significance of left atrial remodelling in competitive athletes. J Am Coll Cardiol 46:690-6.

21. Prinz C, Van Buuren F, Bogunovic N, Bitter T, Faber L, Horstkotte D (2012). In patients with hypertrophic cardiomyopathy myocardial fibrosis is associated with both left ventricular and left atrial dysfunction. Acta Cardiol 67:187-193.

22. Saraiva RM, Demirkol S, Buakhamsri A et al (2010). Left atrial strain measured by twodimensional speckle tracking represents a new tool to evaluate left atrial function. J Am Soc Echocardiogr 2:172-80.

23. Schneider C, Malisius R, Krause K, Bahlmann E, Boczor S (2008). Strain rate imaging for functional quantification of the left atrium: atrial deformation predicts the maintenance of sinus rhythm after catheter ablation of atrial fibrillation. Eur Heart J 20:1397-1409.

24. Sitges M, Teijeira VA, Scalise A et al (2007). Is there an anatomical substrate for idiopathic paroxismal atrial fibrillation? A case control echocardiographic study. Europace 9: 294-8.

25. Sorokin AV, Araujo CG, Zweibel S, Thompson PD (2011). Atrial fibrillation in endurance-trained athletes. Br J Sports Med 45:185-8.

26. Tsai CT, Tseng CD, Hwang JJ et al (2011). Tachycardia of atrial myocites induces collagen expression in atrial fibroblasts through transforming growth factor B1. Cardiovascular Research 89:805-815. 
27. Tsang MY, Barnes ME, Tsang TS (2012). Left atrial volume: clinical value revisited. Curr Cardiol Rep 14:374-80.

28. Turagam M, Velagapudi P, Kocheril A. Atrial fibrillation in athletes (2012). Am J Cardiol 109:296-302.

29. Van Buuren F, Mellwig KP, Faber L et al (2012). The occurrence of atrial fibrillation in former top-level handball players above the age of 50. Acta Cardiol 67:213-220.

30. Wilhelm M, Roten L, Tanner H, Wilhelm I, Schmid JP, Saner H (2011). Atrial remodeling, autonomic tone and lifetime training hours in nonelite athletes. Am J Cardiol 108:580-585. 
Table 1. Clinical characteristics of the studied subjects.

\begin{tabular}{|lccc|}
\hline & Controls & Athletes & p value \\
& $(\mathbf{n = 2 0})$ & $(\mathbf{n}=\mathbf{2 4})$ & \\
\hline Age (years) & $27 \pm 4$ & $28 \pm 4$ & NS \\
Weight (kg) & $72 \pm 8$ & $94 \pm 12$ & $<0.01$ \\
Height $(\mathbf{c m})$ & $175 \pm 15$ & $191 \pm 15$ & $<0.01$ \\
BMI (kg/m $\left.\mathbf{m}^{\mathbf{2}}\right)$ & $23 \pm 2.7$ & $25 \pm 2.6$ & 0.01 \\
BSA (m) & $1.80 \pm 0.12$ & $2.20 \pm 0.17$ & $<0.01$ \\
Heart $\mathbf{~ r a t e ~ ( b p m ) ~}$ & $76 \pm 12$ & $55 \pm 9$ & $<0.01$ \\
\hline
\end{tabular}

Abbreviations: BMI, body mass index; BSA, body surface area

NS, non-significant statistical difference. 
Table 2. Echocardiographic left ventricular and left atrial characteristics of the studied subjects.

\begin{tabular}{|c|c|c|c|}
\hline & $\begin{array}{l}\text { Controls } \\
(\mathrm{n}=\mathbf{2 0})\end{array}$ & $\begin{array}{l}\text { Athletes } \\
(n=24)\end{array}$ & p value \\
\hline LV End-diastolic diameter (cm) & $5.1 \pm 0.3$ & $5.3 \pm 0.6$ & NS \\
\hline LV mass index $\left(\mathrm{g} / \mathrm{m}^{2}\right)$ & $86 \pm 10$ & $116 \pm 21$ & $<0.01$ \\
\hline LV ejection fraction (\%) & $60 \pm 6$ & $57 \pm 5$ & NS \\
\hline LV GLS (\%) & $-20.0 \pm 1.3$ & $-19.0 \pm 1.9$ & NS \\
\hline LA anteroposterior diameter $(\mathbf{c m})$ & $3.5 \pm 0.2$ & $4.1 \pm 0.3$ & $<0.01$ \\
\hline $\mathrm{LA}$ area index $\left(\mathrm{cm}^{2} / \mathrm{m}^{2}\right)$ & $8.6 \pm 1.2$ & $10.1 \pm 1.3$ & $<0.01$ \\
\hline LA volume index $\left(\mathrm{ml} / \mathrm{m}^{2}\right)$ & $24.8 \pm 4.3$ & $35.2 \pm 8.8$ & $<0.01$ \\
\hline LA pre A volume index $\left(\mathrm{mL} / \mathrm{m}^{2}\right)$ & $12.9 \pm 3.4$ & $19.6 \pm 9.2$ & $<0.01$ \\
\hline LA minimal volume index $\left(\mathrm{ml} / \mathrm{m}^{2}\right)$ & $9.5 \pm 3.4$ & $16.6 \pm 8.5$ & $<0.01$ \\
\hline LA emptying fraction & $0.59 \pm 0.23$ & $0.61 \pm 0.35$ & NS \\
\hline $\begin{array}{l}\text { LA emptying fraction in early } \\
\text { diástole }\end{array}$ & $0.45 \pm 0.09$ & $0.46 \pm 0.15$ & NS \\
\hline
\end{tabular}




\begin{tabular}{|c|c|c|c|}
\hline LA A wave VTI (cm) & $4.9 \pm 1.0$ & $4.6 \pm 0.7$ & NS \\
\hline LA active contraction $S V(\mathrm{ml})$ & $7.3 \pm 1.1$ & $6.6 \pm 1.4$ & NS \\
\hline $\begin{array}{l}\text { LA emptying volume in early } \\
\text { diastole }(\mathrm{mL})\end{array}$ & $21.5 \pm 6.2$ & $34.8 \pm 10.2$ & $<0.01$ \\
\hline E wave velocity $(\mathrm{cm} / \mathrm{s})$ & $78 \pm 13$ & $79 \pm 14$ & NS \\
\hline A wave velocity $(\mathrm{cm} / \mathrm{s})$ & $37 \pm 13$ & $42 \pm 7$ & NS \\
\hline EA ratio & $2.1 \pm 0.6$ & $1.9 \pm 0.4$ & NS \\
\hline E wave deceleration time (ms) & $178 \pm 47$ & $205 \pm 51$ & NS \\
\hline A wave duration (ms) & $133 \pm 15$ & $143 \pm 23$ & NS \\
\hline Em septal $(\mathbf{c m} / \mathbf{s})$ & $9.8 \pm 1.6$ & $9.2 \pm 1.8$ & NS \\
\hline Em lateral $(\mathbf{c m} / \mathbf{s})$ & $13.2 \pm 2.6$ & $13.1 \pm 2.1$ & NS \\
\hline $\mathbf{E} / \mathbf{E m}$ ratio & $7.4 \pm 1.1$ & $7.0 \pm 1.0$ & NS \\
\hline LA Sa $(\%)$ & $-14.5 \pm 2.1$ & $-12.2 \pm 2.0$ & $<0.01$ \\
\hline $\operatorname{LASRa}\left(\mathrm{s}^{-1}\right)$ & $-1.87 \pm 0.36$ & $-1.52 \pm 0.59$ & 0.02 \\
\hline LASs (\%) & $36.2 \pm 6.0$ & $35.9 \pm 5.6$ & NS \\
\hline $\operatorname{LASRe}\left(s^{-1}\right)$ & $-2.15 \pm 0.59$ & $-2.18 \pm 0.47$ & NS \\
\hline $\operatorname{LASRs}\left(\mathrm{s}^{-1}\right)$ & $1.65 \pm 0.48$ & $1.64 \pm 0.40$ & NS \\
\hline
\end{tabular}




\begin{abstract}
Abbreviations: Em, early peak velocity of the mitral annulus by Doppler Tissue Imaging; GLS, global longitudinal strain; LA, left atrium; LASa, LA late diastolic (atrial contraction) peak strain; LASRa, LA late diastolic (atrial contraction) peak strain rate; LASRe, LA early diastolic peak strain rate; LASRs, LA peak strain rate during ventricular systole; LASs, LA peak strain during ventricular systole; LV, left ventricle; NS, non-significant statistical difference; SV, stroke volume (planimetry).
\end{abstract}


Table 3. Echocardiographic right ventricular and right atrial characteristics of the studied subjects.

\begin{tabular}{|c|c|c|c|}
\hline & $\begin{array}{l}\text { Controls } \\
(\mathrm{n}=\mathbf{2 0})\end{array}$ & $\begin{array}{l}\text { Athletes } \\
(\mathrm{n}=\mathbf{2 4})\end{array}$ & p value \\
\hline RV basal diameter $\left(\mathrm{mm} / \mathrm{m}^{2}\right)$ & $16.0 \pm 3.1$ & $17.0 \pm 4.1$ & 0.05 \\
\hline $\mathrm{RV}$ fractional area change $(\%)$ & $40 \pm 5$ & $38 \pm 4$ & NS \\
\hline TAPSE $(\mathbf{m m})$ & $28 \pm 3$ & $27 \pm 2$ & NS \\
\hline RV GLS (\%) & $-23.7 \pm 3.3$ & $-22.8 \pm 3.3$ & NS \\
\hline $\mathrm{RA}$ maximal volume index $\left(\mathrm{ml} / \mathrm{m}^{2}\right)$ & $19.0 \pm 5.1$ & $29.0 \pm 8.4$ & $<0.01$ \\
\hline RA pre A volume index $\left(\mathrm{mL} / \mathrm{m}^{2}\right)$ & $14.3 \pm 4.1$ & $20.9 \pm 8.6$ & $<0.01$ \\
\hline RA minimal volume index $\left(\mathrm{ml} / \mathrm{m}^{2}\right)$ & $9.8 \pm 3.0$ & $18.1 \pm 7.9$ & $<0.01$ \\
\hline RA active contraction $\mathrm{SV}(\mathrm{ml})$ & $7.2 \pm 1.1$ & $6.2 \pm 1.3$ & $<0.01$ \\
\hline $\begin{array}{l}\text { RA emptying volume in early diastole } \\
(\mathrm{mL})\end{array}$ & $11.0 \pm 7.6$ & $18.0 \pm 10.8$ & 0.02 \\
\hline RA emptying fraction & $0.48 \pm 0.15$ & $0.39 \pm 0.16$ & NS \\
\hline RA emptying fraction in early diastole & $0.31 \pm 0.17$ & $0.30 \pm 0.17$ & NS \\
\hline RASa (\%) & $-14.2 \pm 1.5$ & $-12.1 \pm 1.8$ & $<0.01$ \\
\hline
\end{tabular}




\begin{tabular}{|lccc|}
\hline $\operatorname{RASRa}\left(\mathbf{s}^{-1}\right)$ & $-2.05 \pm 0.48$ & $-1.32 \pm 0.37$ & $<0.01$ \\
$\operatorname{RASs}(\%)$ & $34.3 \pm 7.5$ & $33 \pm 7.1$ & NS \\
$\operatorname{RASRe}\left(\mathbf{s}^{-1}\right)$ & $-1.98 \pm 0.52$ & $-1.68 \pm 0.50$ & NS \\
& & & \\
$\operatorname{RASRs}\left(\mathbf{s}^{-\mathbf{1}}\right)$ & $2.00 \pm 0.51$ & $1.82 \pm 0.35$ & $\mathrm{NS}$ \\
& & & \\
\hline
\end{tabular}

Abbreviations: GLS, global longitudinal strain; NS, non-significant statistical difference; RA, right atrium; RASa, RA late diastolic (atrial contraction) peak strain; RASRa, RA late diastolic (atrial contraction) peak strain rate; RASRe, RA early diastolic peak strain rate; RASRs, RA peak strain rate during ventricular systole; RASs, RA peak strain during ventricular systole; RV, right ventricle; SV, stroke volume (planimetry); TAPSE, tricuspid annulus plane systolic excursion. 
Table 4. Inter- and intraobserver reproducibility of the speckle tracking measurements

\begin{tabular}{|l|c|c|c|c|}
\hline \multirow{2}{*}{} & \multicolumn{2}{|c|}{ Interobserver agreement } & \multicolumn{2}{c|}{ Intraobserver agreement } \\
\cline { 2 - 5 } & Mean difference & CI 95\% & Mean difference & CI 95\% \\
\hline LASa (\%) & 0.4 & -1.90 to 2.60 & -0.03 & -1.68 to 1.73 \\
LASRa (s $\left.{ }^{-1}\right)$ & -0.05 & -0.50 to 0.60 & -0.04 & -0.33 to 0.24 \\
RASa $(\%)$ & -0.8 & -3.20 to 1.60 & 0.18 & -1.50 to 1.86 \\
RASRa $\left(\mathbf{s}^{-\mathbf{1}}\right)$ & -0.10 & -0.57 to 0.37 & -0.03 & \\
\end{tabular}

Abbreviations: CI, confidence interval; LASa, LA late diastolic (atrial contraction) peak strain; LASRa, LA late diastolic (atrial contraction) peak strain rate; RASa, RA late diastolic (atrial contraction) peak strain; RASRa: LA late diastolic (atrial contraction) peak strain rate. 


\section{Figures title and legend}

Figure 1. Left atrial strain (A) and strain rate (B) curves obtained from $2 \mathrm{D}$ speckle tracking. Arrows indicate LASa and LASRa, respectively.

Figure 2. Relation between the active LA/RA deformation and maximal atrial size in athletes and controls.

Top panels circle: subgroup of athletes with higher LA dilatation and lower LASa (2A), LASRa (2B) and LA active SV by planimetry (2C).

Bottom pannels circle: subgroup of athletes with higher RA dilatation and lower RASa (2D), RASRa (3E) and RA active SV by planimetry (3F).

Figure 3. Modeled relation between pre-A volume, strain and SV with individual data overlaid.

Figure 4. SV reserve to achieve 40\% strain (top); stress-volume relation (middle and bottom). The curve shows the theoretical stress-volume relation, calculated according to equation [27]. 


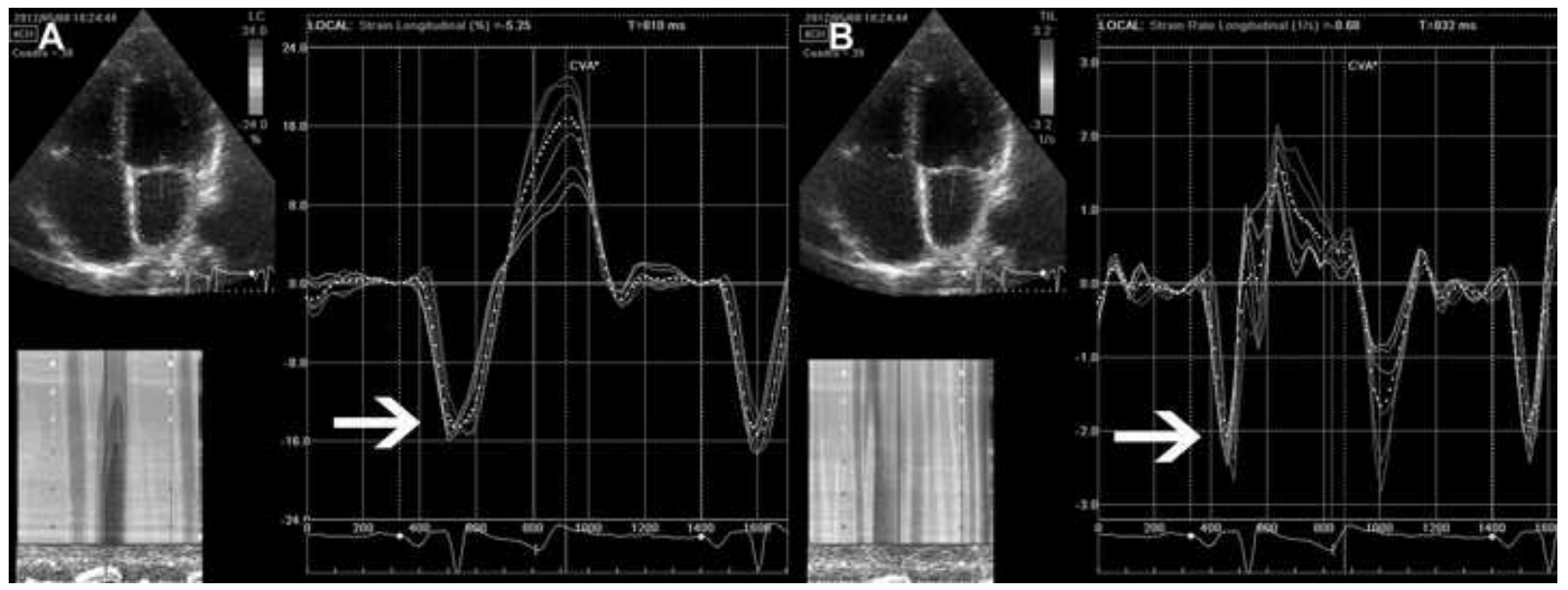



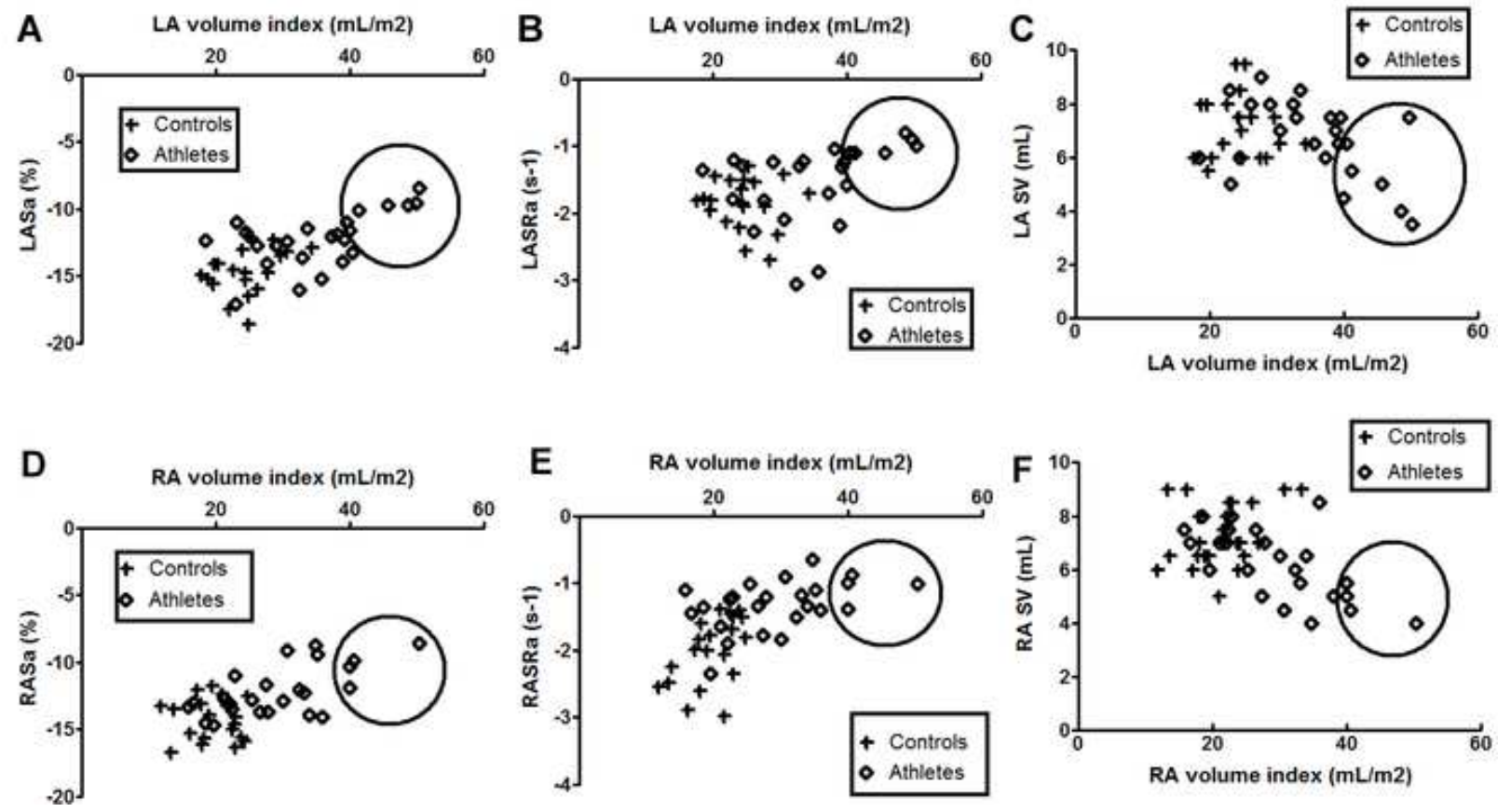


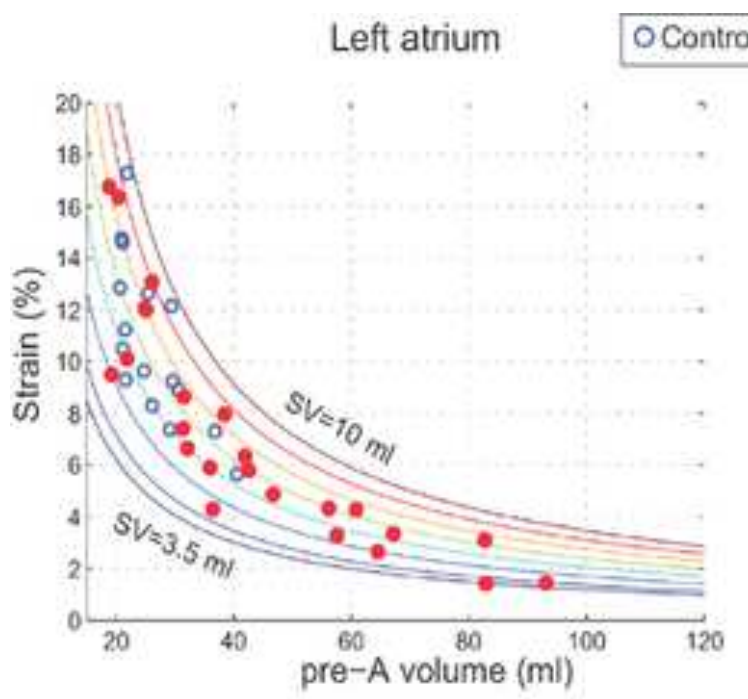

Right atrium
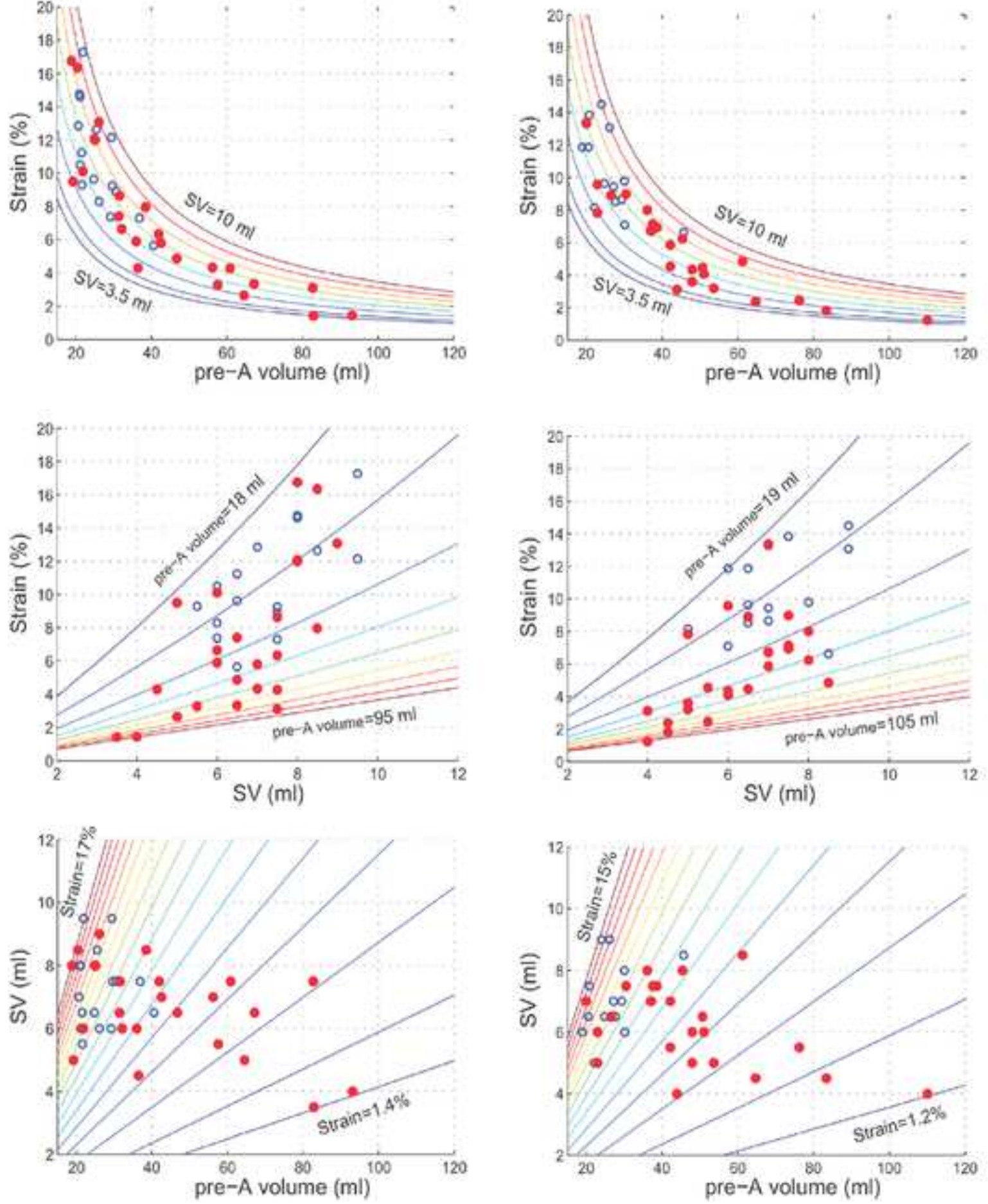

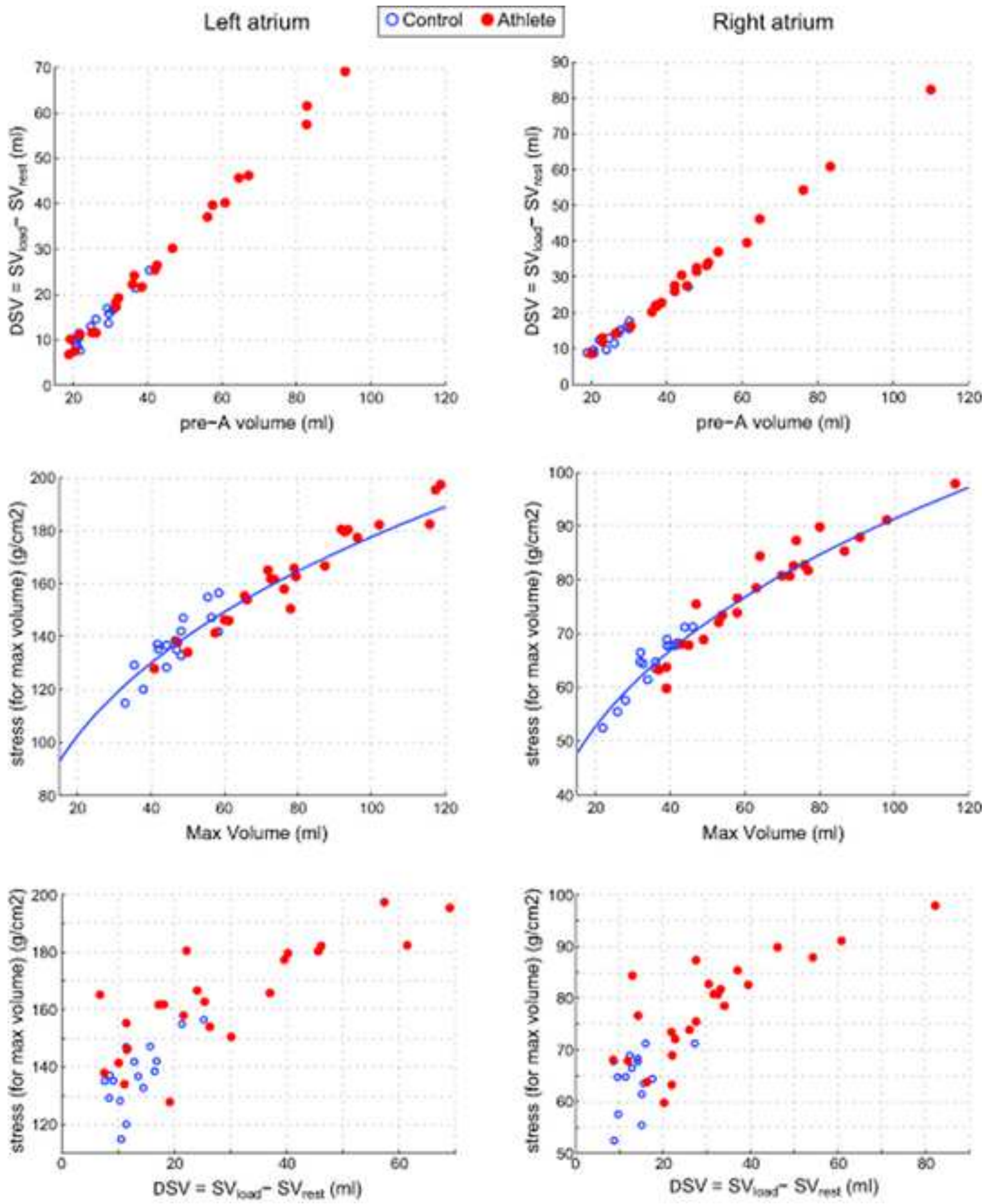